\title{
ON THE DENSITY OF SOME WIENER FUNCTIONALS: AN APPLICATION OF MALLIAVIN CALCULUS
}

\author{
ANTONI SINTES BLANC \\ Dedicated to Professor Pere Menal i Brufal, in memoriam
}

\begin{abstract}
Using a representation as an infinite linear combination of chisquare independent random variables, it is shown that some Wiener functionals, appearing in empirical characteristic process asymptotic theory, have densities which are tempered in the properly infinite case and exponentially decaying in the finite case.
\end{abstract}

\section{Introduction}

Let $F$ be a probability distribution function on $\mathbb{R}$, with characteristic function $c(t):=E(\exp \{i t X\}), X$ being a real random variable with distribution function $\mathbf{F}$.

Let $X_{1}, X_{2}, \ldots$ be a sequence of independent copies of $X$ defined on some probability space $(\Omega, \mathcal{F}, \mathbb{P})$, and $\boldsymbol{F}_{n}$ the empirical distribution function of the first $n$ variables.

The empirical characteristic function $c_{n}(t)$ is the (random) characteristic function of $F_{n}$, and has been used in several statistical applications since at least Cramer's famous book.

In the late 70's a systemalic study of its properties and statistical applications was initiated by Feuerverger and Mureika. In particular they proved the first linuit theorems for the empirical characteristic process $Y_{n 1}(t):=\sqrt{n}\left(c_{n}(t)-c(t)\right)$, under strong moment conditions on $\mathbb{F}$, see [2].

In 1981 M. Marcus, [3], found necessary and sufficient conditions for the processes $\left\{Y_{n}(t)\right\}_{n-1}^{\infty}, t \in\left[T_{1}, T_{1}\right]$, to werkly converge to a limit process of the following form

$$
Y(t):=\int_{-\infty}^{+\infty} \exp \{i t x\} d \mathbb{B}(\mathbb{F}(x))
$$


where $\mathbb{B}$ is Brownian bridge on $[0,1]$, as $C\left[T_{1}, T_{2}\right]$ valued random elements.

The same year S. Csörgó, [1, Theorem 3], obtained strong approximations for $Y_{n}(t)$, under the assumption

$$
h(x)[1-F(x)+F(-x)]=O(1), \quad \text { as } x \text { goes to }+\infty
$$

where $h$ is a continuous function on $(0,+\infty)$ and $h(x) x^{-\alpha}$ increases to $+\infty$ as $x$ increases to $+\infty$, for some positive $\alpha$.

From this strong approximations S. Csörgó derived rates of convergence for several functionals of the paths of the empirical claracteristic process, under the further assurnption of existence and boundedness of a density for the limit functional.

Here we want to study the following one which is useful in testing for symmetry of $F$, namely

$$
\mathbf{T}:=\int_{T_{1}}^{T_{2}}[\operatorname{Im}(\mathbf{Y}(t))]^{2} d \mathrm{H}(t)
$$

where $\mathrm{H}$ is a given distribution function with support in $\left[T_{1}, T_{2}\right]$, and $-\infty<T_{1}<T_{2}<+\infty$.

The main result in the present work is that with great generality, $\mathbb{T}$ has a density which in fact satisfies much stronger boundedness conditions than those needed for Csörgó's rates of convergence to hold true. It is the following

Theorem. Assume that the randon variable $\mathrm{T}$, as defined above, is non degenerate (this is a condition on both, $\mathbb{F}$ and $\boldsymbol{H}$ ). Then $\mathbb{T}$ has a smooth density, which is either a tempered function or an exponentially decaying one (at infinity).

The main ingredients of the proof are Malliavin calculus, Cauchy's formula and Fourier transform. In the next paragraph we recall, very briefly, the principal definitions and results we are going to use, and give some references where completc and detailed expositions can be found. Finally, in paragraph 3 we give the proof of the theorem.

\section{The tools}

Let $\mathbb{L}^{2}[0,1]$ be the Hilbert space of square integrable functions with respect to the Lebcsgue measure on the Borel $\sigma$-field, $B$, in the unit interval $[0,1]$. 
For $h \in \mathbb{L}^{2}[0,1]$, denote $\boldsymbol{W}(h)$ the Wiener integral of $h$ with respect to the Wiener process, $W$, on $[0,1]$. We think of $W$ as a Gaussian orthogonal measure on the space $([0,1], \mathcal{B}, \lambda)$, it is, a zero mear Gaussian process $\{W(B): B \in \mathcal{B}\}$ defined on some probability space $(\Omega, \mathcal{F}, P)$, with covariance function given by $E\left(W\left(B_{1}\right) W\left(B_{2}\right)\right)=\lambda\left(B_{1} \cap B_{2}\right)$, where $\lambda$ is Lebesgue measure on $[0,1]$.

Around $1950 \mathrm{~K}$. Ito showed that each square integrable functional $F \in \mathbb{L}^{2}(\Omega, \mathcal{F}, P)$ can be developed in the form

$$
F=E(F)+\sum_{m=1}^{\infty} I_{m}\left(f_{m}\right)
$$

where $I_{m}\left(f_{m}\right)$ are multiple Ito-Wiener integrals of the (deterministic) functions $f_{m} \in \mathbb{L}^{2}\left([0,1]^{m}, \mathcal{B}^{m}, \lambda^{m}\right)$. In particular $I_{1}\left(f_{1}\right)=\mathbb{W}\left(f_{1}\right)$. This expansion is sometimes known as the Wiener chaos decomposition of $F$.

Stroock's formula identifies the kernels, $f_{m n}$, in terns of iterated Malliavin derivatives of $F$ :

$$
f_{m}=(m !)^{-1} E\left(D^{m} F\right)
$$

For $F \in \mathbb{L}^{2}(\Omega, \mathcal{F}, P)$ and $h \in \mathbb{L}^{2}[0,1]$, the Malliavin derivative of $F$ in the $h$ direction, $D_{h l} F$, can be defined as

$$
D_{h} F:=\sum_{m=1}^{\infty} m\left\langle h, \quad I_{m n-1}\left(f_{m}\left(t_{1}, \ldots, t_{m-1}, *\right)\right)\right\rangle
$$

if the series converges in $\mathbf{L}^{2}(\Omega, \mathcal{F}, P)$, where $\langle$,$\rangle is inner product. All$ this (and much more) can be found in $[\mathbf{4}]$ and $[\mathbf{5}]$.

To apply this theory to our functional $\mathrm{T}$, we write it down in terrns of $W$ as follows:

$$
\mathrm{T}=\int_{T_{1}}^{T_{2}}\left[W\left(h_{t}\right)\right]^{2} d \mathrm{H}(t)
$$

where $h_{t}(y):=\sin \left(t F^{-1}(y)\right)-\operatorname{Im}(c(t))$, for $y \in[0,1], c(t)$ being the characteristic function of $F$, i.e. $c(t):=E(\exp \{i t \mathbf{X}\})$, where $\mathbf{X}$ is a $\mathbf{F}$ distributed random variable.

Let us calculate the Malliavin derivatives

$$
\begin{aligned}
D\left[W\left(h_{t}\right)\right]^{2} & =2 \mathbb{W}\left(h_{t}\right) h_{t} \\
D^{2}\left[\mathbb{W}\left(h_{t}\right)\right]^{2} & =2 h_{t} \otimes h_{t}
\end{aligned}
$$

and the derivatives of order greater or equal than 3 are all zero. 
Hence; by linearity

$$
D^{2} \mathbb{T}=\int_{T_{1}}^{T_{2}} 2 h_{t} \otimes h_{l} d \mathbb{H}(t)
$$

and from Stroock's formula we get.

$$
T=E(T)+I_{2}\left(\int_{T_{1}}^{T_{2}} h_{t} \otimes h_{t} d \mathfrak{H}(t)\right)
$$

Now we recall some more definitions and results that will also be needed in the next paragraph.

A function $f$ on $\mathbb{R}$ is called a tempered function if it is a smooth function and for each $N \in \mathbb{N}$ (the set of natural numbers)

$$
\sup _{p \leq N} \sup _{x \in \mathbb{R}}\left(1+x^{2}\right)^{N}\left|f^{(p)}(x)\right|<+\infty
$$

where $f^{(p)}$ is the $p$-th derivative of $f$ :

Let $S$ denote the space of tempercd functions on $\mathbb{R}$. The Fouricr transform of a function $f \in S$ is defined as

$$
\hat{f}(t):=(2 \pi)^{-\frac{1}{2}} \int_{-\infty}^{+\infty} \operatorname{cxp}(-i t x) f(x) d x
$$

It turns out that this transformation defines a continuous one-to-one mapping of $S$ onto $S$, whose inverse is also continuous. Moreover, it has period 4 , due to the inversion formula which is fundamental

$$
f(x)=(2 \pi)^{-\frac{1}{2}} \int_{-\infty}^{+\infty} \exp (i t x) \hat{f}(t) d t
$$

A very good reference for this is $[6]$.

\section{Proof of the theorem}

The idea of the proof is quite simple: to show that the characteristic function of $T$ is a tempered function in the properly infinite case, and direct calculation in the finite case (distinction of the two cases will be clear in a moment).

Let us go back to Stroock's representation of $\mathbf{T}$, and recall the explicit form of the kernel in the double Ito-Wiener integral there. 
It is known that such a kemel can be developed in a $\mathbb{L}^{2}$-convergent series.

$$
K^{\prime}(r, s)=\sum_{i=1}^{\infty} \lambda_{i} e_{i}(r) e_{i}(s)
$$

where $\lambda_{i}$, (respectively $e_{i}(\cdot)$ ), are the eigenvalues, (respectively eigenvectors) of the following self-adjoint non-negative integral operator

$$
\phi(\cdot) \longmapsto \int_{0}^{1} K(\cdot, s) \phi(s) d s
$$

on $\mathbb{L}^{2}[0,1]$, the $e_{i}(\cdot)$ 's can be assumed to be a complete orthonormal system in $\mathbb{L}^{2}[0,1]$, and $\sum_{i=1}^{\infty} \lambda_{i}<+\infty$.

It is also well known that for $h \in \mathbb{L}^{2}\{0,1\}$, with $\|h\|_{2}=1$

$$
I_{2}(h \otimes h)=\mathbb{W}(h)^{2}-1
$$

and combination of this facts leads us to the representation we had in mind, namely

$$
\boldsymbol{T}=E(\mathbb{T})+\sum_{i=1}^{\infty} \lambda_{i}\left(W\left(e_{i}\right)^{2}-1\right)=\sum_{i=1}^{\infty} \lambda_{i} W\left(e_{i}\right)^{2}
$$

with $\sum_{i=1}^{\infty} \lambda_{i}<+\infty$.

We remark here that from this representation it is clear that $\pi$ is non-degenerate iff Stroock's kemel, $D^{2} \mathrm{~T}$, is non-degenerate.

To proced we distinguish two cases: the one where only a finite number of the $\lambda_{i}$ are non zero, and the one where there are infinitely many non zero. We treat only the second case, as the first one is very elementary. So, to finish the proof of the theorem it is enough to prove next. lemma,

Lemma. Let $\left\{\boldsymbol{X}_{i}\right\}_{i=1}^{\infty}$ be independent identically distributed random variables with a conmon chi-square distribution, i.e. a distribution with densily $x^{-\frac{1}{2}} \cdot \exp \left(-\frac{x_{2}}{2}\right) 1_{(0, \infty)}(x)$.

Let $\left\{\lambda_{i}\right\}_{i=1}^{\infty}$ be a nonincreasing sequence of positive real numbers such that $\sum_{i=1}^{\infty} \lambda_{i}<+\infty$.

Then the random variable $\boldsymbol{J}:=\sum_{i=1}^{\infty} \lambda_{i} X_{i}$ has a tempered probability density function.

Proof: It is well known that the characteristic function of the random variable $\rightarrow$ is

$$
\hat{f}(t)=\prod_{k=1}^{\infty}\left(1-2 \lambda_{k} i t\right)^{-\frac{1}{2}}
$$


It is easily secn that this function possesses an analytic contimuation on the strip $-\left(4 \lambda_{1}\right)^{-1}<\operatorname{Im}(z)<\left(4 \lambda_{1}\right)^{-1}$, which is of the same form, i.e.

$$
\hat{f}(z)=\prod_{k=1}^{\infty}\left(1-2 \lambda_{k} i z\right)^{-\frac{1}{2}}
$$

where $i:=\sqrt{-1}$. Observe that for $z$ in this strip

$$
\frac{1}{2}<\operatorname{Re}(1-2 i \lambda z)<\frac{3}{2}
$$

so that we can use the branch of $\sqrt{z}$ which coincides with the usual square root function on the positive real numbers, and we can apply Theorem (15.6) from [7], (here we use the hypothesis $\sum_{i=1}^{\infty} \lambda_{i}<+\infty$ ).

For $t>0$ apply Cauchy's formula to the rectangle $\Gamma$ defined by the points $(t / 2) \mp i\left(4 \lambda_{1}\right)^{-1}, 2 t \mp i\left(4 \lambda_{1}\right)^{-1}$, to get

$$
\hat{f}^{(p)}(t)=(p !) \sum_{j=1}^{4} \int_{\Gamma_{j}} \frac{\hat{f}(z)}{(z-t)^{p+1}} d z
$$

where $\left\{\Gamma_{j}\right\}_{j=1}^{4}$ are the four sides of the rectangle $\Gamma$.

To bound this integrals we use the fact that for each natural number $M \in \mathbb{N}$

$$
\begin{aligned}
& \sup _{|s| \leq\left(4 \lambda_{1}\right)^{-1}}|\hat{f}((t / 2)+i s)| \leq\left(\prod_{k=1}^{M}\left((1 / 4)+\lambda_{k}^{2} t^{2}\right)\right)^{-\frac{2}{4}} \\
& \sup _{(t / 2) \leq|s| \leq 2 t}\left|\hat{f}\left(s+i\left(4 \lambda_{1}\right)^{-1}\right)\right| \leq\left(\prod_{k=1}^{M}\left(1+\lambda_{k}^{2} t^{2}\right)\right)^{-\frac{1}{4}}
\end{aligned}
$$

For instance the integral on the left side, let us say $\Gamma_{1}$, of the rectangle $\Gamma$, can be bounded as follows

$$
\begin{aligned}
\int_{-\left(4 \lambda_{1}\right)^{-1}}^{\left(4 \lambda_{1}\right)^{-1}}|\hat{f}((t / 2)+i s)| \mid((t / 2) & -i s)\left.\right|^{-1-p} d s \leq \\
& \leq 2^{p} \lambda_{1}^{-1} t^{p+1}\left(\prod_{k=1}^{M}\left((1 / 4)+\lambda_{k}^{2} t^{2}\right)\right)^{-\frac{1}{4}}
\end{aligned}
$$

where we used (1); and the same type of bound is obtained for the integral on the right side, $\Gamma_{3}$, of $\Gamma$. In a similar way the bound in (2) is used to treat the integrals on the horitzontal sides of the rectangle, $\Gamma_{2}$ and $\Gamma_{4}$. And this proves the lemma in the case $t>0$. 
For $t<0$, with small changes, all works the same way, and for $t=0$ there is no probiem at all. Thus the lemma is proved.

Remark. In the above proof we could have used the asymptotic representation obtained in [8], instead of our lemma. It is clear from [8] that the density $f(x)$ is exponentially decaying at infinity, even in the properly infinite case. However, it is not clear how can we get the temperate character of $f$ from Zolotarev's representation, without further work. In that point our proof seems to be shorter and clearer.

Acknowledgments. I want to thank Professors Paul Malliavin and David Nualart for their kind attention and help on some problems rclated to the subject of this paper. Thanks also to Professor Evarist Gine, to point me out reference [8].

\section{References}

1. S. Csörcó, Limit behavior of the empirical characteristic function, The Annals of Probability 9, no. 1 (1981), 130-144.

2. A. Feuerverger and R. A. Mureika, The empirical characteristic function and its applications, Ann. Statist. 5 (1977), 88-97.

3. M. B. MArcus, Weak convergence of the empirical characteristic function, Ann. Probability 9 (1981), 194-201.

4. D. NUALART AND M. ZAKAI, Gencralized stochastic integrals and the Malliavin calculus, Probab. Theory Rel. Fields 73 (1986), $255-280$.

5. D. Nualart and M. ZaKal, Generalized multiple stochastic integrals and the representation of Wiener functionals, Stochastics 23 (1988), 311-330.

6. W. RUdin, "Functional Analysis," McGraw-Hill, 1973.

7. W. RudiN, "Real and complex analysis:" McGraw-Hill, 1970.

8. V. M. Zolotarev, Concerning a certain probability problem, Theory of Probab. Appl. 6 (1960), 201-203.

Deparlament de Matemátiques

Universitat Autònoma de Barcelona

08193 Bellaterra (Barcelona)

SPAIN

Rebut el 18 de Desembre de $\$ 991$ 